\title{
Perfil ocupacional del consumidor de drogas
}

\author{
María Elena Riveros Espiñeira
}

\begin{abstract}
- Resumen
El presente estudio profundiza en el conocimiento de los usuarios de programas de tratamiento y rehabilitación por consumo de drogas mediante la exploración de la variable historia de desempeño ocupacional. Su hipótesis central es que el sujeto consumidor de drogas que ingresa a un programa de tratamiento y rehabilitación presenta una historia ocupacional desequilibrada o disfuncional que estaría asociada a ese consumo.
\end{abstract}

Para el desarrollo de la investigación se utilizó el instrumento Entrevista Histórica de Funcionamiento Ocupacional (OPHI-II). Se realizaron 28 entrevistas que permitieron construir un perfil ocupacional del consumidor de drogas. Los hallazgos indican que existe cierto grado de disfunción ocupacional en las tres escalas que conforman el instrumento (identidad ocupacional; competencia ocupacional; ambientes de comportamiento ocupacional), lo que a su vez es posible de asociar con el consumo de drogas.

Estos hallazgos respecto al perfil ocupacional del consumidor de drogas son de gran utilidad, pues permiten identificar algunos de los componentes que debieran formar parte de un programa de prevención que sea eficaz, es decir, capaz de evitar o retrasar el inicio del consumo de drogas.

Lo anterior debiera conducir a un posterior análisis crítico de las políticas y programas en prevención actualmente en curso y al diseño preliminar de una estrategia de prevención que dé mejor respuesta a las necesidades de los destinatarios de las mismas, materia que excede los objetivos del estudio, pero que parece importante explicitar.

Palabras clave: consumo de drogas; perfil ocupacional; prevención

\begin{abstract}
- Abstract
The aim of this research is to study occupational performance history to obtain further knowledge about persons that are in treatment and rehabilitation programs for drug abuse. The assumption is that persons drug abusing, that go into treatment and rehabilitation, have an imbalance in their previous occupational performance history that can be related to the abuse.

The Occupational Performance History Interview (OPHI-II) was used in this study covering twenty eight participants. A first tentative occupational profile of a drug abuser was constructed from the data gathered. Imbalance in occupational identity, occupational competence and occupational performance environments (the three scales used in the OPHI-II instrument) is possibly associated with drug abuse according to the findings.
\end{abstract}


An occupational profile, as defined in this study, could be useful to identify components of an effective preventive drug abuse program, which may allow to avoid or delay drug abuse. Furthermore, the author believes that this findings could be useful in a critical analysis of policies and programs currently being used by the state, however, this is a matter that exceeds the scope of the study. 


\begin{abstract}
Antecedentes
Este Estudio ha sido realizado en el marco del Proyecto de Evaluación encargado por CONACE a la Escuela de Salud Pública de la Universidad de Chile (1). El Consejo Nacional de Control de Estupefacientes (CONACE) es la entidad responsable de asesorar al Gobierno de Chile en la formulación, financiamiento y coordinación de políticas y planes destinados al control del consumo de diferentes tipos de drogas.
\end{abstract}

Este trabajo forma parte de la Tesis para optar al grado de Magíster en Salud Pública (2) y fue presentado en el mes de junio en el V Congreso Latinoamericano de Terapia Ocupacional realizado en Foz de Iguazú, Brasil, 2003. Participaron en él las siguientes personas:

\title{
Alumnos de cuarto año de Terapia Ocupacional de la Universidad de Chile:
} Rodrigo Cubillos; Elba Lizama; Marta Martínez

Terapeutas Ocupacionales: Jeannette Vargas; Ana Valdebenito.

\section{- Revisión de literatura}

Una revisión de la literatura reveló la ausencia de investigación que describa el perfil del consumidor de drogas desde una perspectiva ocupacional. El conocer este perfil puede sugerir caminos que permitan apoyar a los sujetos que se enfrentan al problema del consumo de drogas. Este nuevo conocimiento también puede proveer una orientación acerca de cómo deben ser diseñados los programas y estrategias de prevención del consumo de drogas considerando las características ocupacionales de los sujetos hacia quienes van destinados.

La actividad forma parte de la vida los seres humanos. Una actividad debe tener propósito y proporcionar significado a la persona que la realiza para favorecer el desarrollo de destrezas y su adaptación al ambiente. La actividad con propósito (ocupación) incluye componentes interpersonales y ambientales y puede ser utilizada para prevenir y mediar la disfunción, produciendo la máxima adaptación posible (으).

La vida de cada persona está llena de actividades, las que pueden perseguir diferentes propósitos. El compromiso en una actividad no es un acto pasivo. Se deben realizar elecciones, utilizar segmentos del cuerpo, solucionar problemas, interactuar con otras personas o con el ambiente físico y reaccionar al éxito, fracaso o quizá frustración y ansiedad.

La actividad también tiene un aspecto temporal. Dentro de cada período de veinticuatro horas, el tiempo se divide participando en una variedad de actividades que apoyan la función y competencia de cada persona en las áreas de autocuidado, productividad y tiempo libre. Las personas experimentan por sí mismas y conocen sus capacidades y limitaciones por medio de la experiencia del quehacer diario $(\underline{3}, \underline{5})$.

Los roles vitales que una persona espera satisfacer (por ejemplo, estudiante, madre, padre, trabajador) influyen sobre las actividades elegidas y el tiempo que se destina a cada una de ellas, de acuerdo a su realidad y sentido otorgado. 
La ocupación es el foco central para la ciencia de la ocupación y ha sido propuesta como fundamento para la Terapia Ocupacional $(\underline{10}, 11,12)$. Uno de sus conceptos básicos es el equilibrio ocupacional, que ha sido definido como el balance entre el tiempo destinado al trabajo, juego y descanso en la vida cotidiana.

El significado ocupacional es otro concepto básico. Los individuos experimentan y expresan significado en sus vidas a través de las ocupaciones que realizan.

Así, el comportamiento ocupacional representa un aspecto central de la salud, el bienestar, el desarrollo y el cambio. De acuerdo al Modelo de Ocupación Humana ( $\underline{3}$ ), base teórica de la Entrevista Histórica de Funcionamiento Ocupacional, el comportamiento ocupacional es el resultado de una interrelación entre tres componentes (volición, habituación y ejecución) y las circunstancias del medio ambiente. Este Modelo considera a los seres humanos como sistemas, abiertos y dinámicos, que se organizan a sí mismos, que siempre están en desarrollo y que cambian con el tiempo, es decir, se caracterizan por ser abiertos y dinámicos. El comportamiento ocupacional que se esté llevando a cabo subyace a esta autoorganización. Es decir, conforme los seres humanos participen del trabajo, el juego y las actividades de la vida diaria y autocuidado, descubren, mantienen, refuerzan, conforman y modifican sus propias capacidades, creencias y disposiciones o inclinaciones. Por lo tanto, es fundamental conocer y comprender la historia de vida ocupacional de una persona, porque esa historia ha conformado lo que esta persona es y ha creado una trayectoria continua de vida en la cual se encuentra y se identifica en el presente.

El conocer y examinar las necesidades, la historia ocupacional, los roles vitales, los valores culturales, la configuración de la actividad (organización del tiempo utilizado en actividades diarias específicas) y la capacidad alcanzada por las diferentes áreas (neuromuscular, de integración sensorial, cognitiva, psicológica y social), permitirían planificar acciones que den mejor resultado, según las características de los usuarios de los programas de tratamiento y rehabilitación por consumo de drogas (므).

Dado que estos programas de tratamiento y rehabilitación no logran la eficacia deseada, una opción mucho más prometedora y con una mejor relación costo-beneficio es la prevención. El desarrollo de programas de prevención eficaces dirigidos a niños, adolescentes y jóvenes se vuelve indispensable cuando se quiere evitar que el consumo de drogas se convierta en un problema social de incalculable magnitud $(\underline{6}, \underline{7}, \underline{8}, \underline{9})$.

El estudio del desempeño ocupacional de los usuarios y su caracterización ocupacional, permitirá identificar posibles patrones similares en los sujetos consumidores de drogas e incluirlo como una variable a considerar desde la prevención primaria. El propósito es identificar algunos de los componentes que debieran formar parte de un programa de prevención que sea eficaz, es decir, capaz de evitar o retrasar el inicio del consumo de drogas.

La presente tesis plantea que el sujeto en estudio ha tenido pocas oportunidades a lo largo de su vida de realizar actividades con propósito y significado para sí mismo (ocupaciones) que le permitan desarrollar sentido de eficacia, destrezas y adaptarse al ambiente. Esta persona no logra organizar y ejecutar una rutina en que combine en forma equilibrada actividades de autocuidado, productividad y tiempo libre (equilibrio en el desempeño ocupacional) presentando mayor riesgo de tener conductas 
desadaptativas, en las que se incluye el consumo de drogas. La actividad con propósito (ocupación) incluye componentes interpersonales, ambientales, culturales, sociales e históricos. La oportunidad de realizar actividades con propósito puede ayudar a prevenir y mediar la disfunción, produciendo la máxima adaptación $(\underline{3}, \underline{5})$

\section{- Método}

Esta investigación consiste en un estudio cualitativo, de carácter exploratorio y descriptivo, respecto a la historia ocupacional de sujetos que se encuentren actualmente en programas de tratamiento y rehabilitación en drogas. Se entrevistó a 28 participantes utilizando el instrumento Entrevista Histórica de Funcionamiento Ocupacional (OPHI-II).

Los hallazgos serán complementados con el perfil del usuario, con miras a levantar una propuesta de intervención en prevención primaria en drogas que dé mejor respuesta a las necesidades y perfil de los destinatarios.

La unidad de estudio la conforman los individuos beneficiarios de un plan de tratamiento y rehabilitación de drogas financiado por el convenio CONACE-MINSALFONASA.

Cabe señalar que por tratarse de un estudio de carácter cualitativo se aplicó el criterio de saturación para determinar el tamaño de la muestra final. La saturación de la información se refiere a un tipo de técnica de la metodología cualitativa que permite determinar el momento en que un conjunto de datos recogidos, siempre de la misma manera, comienza a repetirse en término de los puntajes asignados a cada ítem o reactivo y, por lo tanto, no aporta nueva información a la ya encontrada. La saturación se producirá cuando al agregarse un sujeto más al estudio no ocurran modificaciones significativas a los puntajes y promedios ya obtenidos, de manera que éstos se empiecen a repetir, tanto a nivel de los reactivos que conforman cada una de las escalas como en la totalidad de la misma, lo que indicará que se finaliza la toma de la muestra. 


\section{- Recolección de información}

Para el estudio de la historia de desempeño ocupacional se aplicó el Instrumento llamado Entrevista Histórica de Desempeño Ocupacional (OPHI-II), adaptado a los objetivos del presente Estudio (4).

El instrumento consiste en: a) una entrevista semiestructurada, b) tres escalas para calificar la información obtenida en la entrevista, y c) un formato para registrar datos cualitativos (es decir, narrativos). La entrevista está diseñada para reunir los datos esenciales sobre la historia de vida ocupacional del cliente $(\underline{13}, \underline{14})$. La entrevista semiestructurada está organizada en las siguientes áreas temáticas:

- Elecciones de Actividad/ Ocupación

- Eventos Críticos en la Vida

- Rutina Diaria

- Roles Ocupacionales

- Ambientes de Comportamiento Ocupacional

La segunda parte del OPHI-II está formada por tres escalas de calificación, éstas son:

- Escala de Identidad Ocupacional

- Escala de Competencia Ocupacional

- Escala de Ambientes de Comportamiento Ocupacional

La entrevista implica la conducción de la conversación (o serie de conversaciones) con la persona para aprender acerca de su historia de vida ocupacional. Antes de hacer la entrevista, el entrevistador debe reunir cualquier información relevante acerca del cliente, la cual enriquecerá la entrevista. En el caso particular de este estudio, el entrevistador aplicó previamente el Instrumento de "Caracterización del Perfil del Usuario" que reúne antecedentes sociodemográficos; situación laboral previa al consumo; situación legal; motivo de ingreso al centro; historia de consumo; historia de tratamiento.

El segundo componente del OPHI-II son las escalas de calificación. Hay tres escalas que corresponden al contenido de la entrevista: Identidad Ocupacional; Competencia Ocupacional; Ambientes de Comportamiento Ocupacional.

Las dos primeras incluyen reactivos relativos a la persona entrevistada. La escala de Ambientes de Comportamiento Ocupacional se enfoca en el impacto de los ambientes físicos y sociales del trabajo, el hogar y el tiempo libre, que puede registrarse independientemente de lo anterior.

El OPHI-II utiliza una escala de cuatro puntos. La asignación de una calificación requiere que el entrevistador utilice la información obtenida de la entrevista para hacer un juicio de la pregunta que se califica. Este juicio implica entender claramente la pregunta a ser evaluada, el sistema de calificación y la información obtenida en la entrevista.

Se desarrolló una capacitación para la aplicación del Instrumento que evalúa la historia ocupacional. Para ello, se capacitó a tres alumnos de la carrera de Terapia Ocupacional de la Universidad de Chile, que participan del Programa Ayudante Alumno que 
desarrolla la Facultad de Medicina. La capacitación de estos alumnos tuvo aproximadamente un mes de duración, dividida en varias etapas.

El estudio piloto del OPHI-II se efectuó en la Región Metropolitana en centros seleccionados al azar entre el conjunto de establecimientos que tienen convenio suscrito con CONACE/MINSAL/FONASA, pero que no fueron seleccionados en la muestra global del estudio. Consistió en siete entrevistas de historia ocupacional, las que fueron analizadas posteriormente. El análisis se realizó en cinco reuniones, con un total aproximado de quince horas de trabajo. La metodología a seguir fue la lectura de las respuestas a cada una de las preguntas y la discusión de la calificación de ésta en una escala con el fin de "calibrar" los criterios aplicados por los diferentes encuestadores. Paralelamente se fue realizando una descripción del perfil ocupacional del sujeto entrevistado, para finalmente otorgarle una calificación o puntaje de funcionamiento ocupacional.

\section{- Resultados}

Al momento de realizar un primer análisis de los resultados se contaba con 25 entrevistas terminadas.

\section{Hoja Resumen OPHI-II ( $n=25$ entrevistas)}

\begin{tabular}{|c|c|c|c|c|c|c|}
\hline Escala de Identidad Ocupacional & 1 & 2 & 3 & 4 & $\begin{array}{c}\text { Total Suma } \\
\text { Reactivos } \\
\text { Escala }\end{array}$ & $\begin{array}{c}\text { Puntaje } \\
\text { Acumulado } \\
\text { (x) }\end{array}$ \\
\hline Tiene metas personales y proyectos & 3 & 36 & 12 & 0 & 51 & 2.0 \\
\hline Identifica un estilo de vida ocupacional & 3 & 32 & 18 & 0 & 53 & 2.1 \\
\hline Espera el éxito & 7 & 28 & 12 & 0 & 47 & 1.9 \\
\hline Acepta responsabilidades & 7 & 26 & 15 & 0 & 48 & 1.9 \\
\hline Valora habilidades v limitaciones & 2 & 26 & 30 & 0 & 58 & 2.3 \\
\hline Tiene compromisos y valores & 5 & 26 & 21 & 0 & 52 & 2.1 \\
\hline Reconoce identidades y obligaciones & 3 & 32 & 18 & 0 & 53 & 2.1 \\
\hline Tiene intereses & 0 & 28 & 33 & 0 & 61 & 2.4 \\
\hline Se sintió eficaz (pasado) & 5 & 18 & 33 & 0 & 56 & 2.2 \\
\hline $\begin{array}{l}\text { Encontró sentido / satisfacción en su } \\
\text { estilo de vida (pasado) }\end{array}$ & 5 & 20 & 30 & 0 & 55 & 2.2 \\
\hline Hizo elecciones ocupacionales (pasado) & 3 & 40 & 6 & 0 & 49 & 2.0 \\
\hline $\begin{array}{l}\text { Escala de Competencia } \\
\text { Ocupacional }\end{array}$ & 1 & 2 & 3 & 4 & $\begin{array}{l}\text { Total Suma } \\
\text { Reactivos } \\
\text { Escala }\end{array}$ & $\begin{array}{c}\begin{array}{c}\text { Puntaje } \\
\text { Acumulado }\end{array} \\
(\mathbf{x}) \\
\end{array}$ \\
\hline Mantiene un estilo de vida satisfactorio & 6 & 34 & 6 & 0 & 46 & 1.8 \\
\hline $\begin{array}{l}\text { Cumple con las expectativas de sus } \\
\text { roles }\end{array}$ & 6 & 28 & 15 & 0 & 49 & 2.0 \\
\hline
\end{tabular}




\begin{tabular}{|l|c|c|c|c|c|c|}
\hline Trabaja hacia metas personales & 6 & 36 & 3 & 0 & 45 & 1.8 \\
\hline $\begin{array}{l}\text { Cumple con los estándares de } \\
\text { rendimiento personales }\end{array}$ & 3 & 30 & 21 & 0 & 54 & 2.2 \\
\hline $\begin{array}{l}\text { Organiza su tiempo para cumplir } \\
\text { responsabilidades }\end{array}$ & 4 & 40 & 3 & 0 & 47 & 1.9 \\
\hline Participa en intereses & 4 & 34 & 12 & 0 & 50 & 2.0 \\
\hline Cumplió con sus roles (pasado) & 10 & 24 & 9 & 0 & 43 & 1.7 \\
\hline Mantuvo hábitos (pasado) & 8 & 16 & 27 & 0 & 51 & 2.0 \\
\hline Logró satisfacción (pasado) & 4 & 30 & 18 & 0 & 52 & 2.1 \\
\hline \hline \multicolumn{1}{|c|}{ Escala de Ambientes de } & $\mathbf{1}$ & $\mathbf{2}$ & $\mathbf{3}$ & $\mathbf{4}$ & $\begin{array}{c}\text { Total Suma } \\
\text { Reactivos } \\
\text { Escala }\end{array}$ & $\begin{array}{c}\text { Puntaje } \\
\text { Acumulado } \\
(\mathbf{x})\end{array}$ \\
\hline $\begin{array}{l}\text { Formas ocupacionales de vida en el } \\
\text { hogar }\end{array}$ & 8 & 22 & 18 & 0 & 48 & 1.9 \\
\hline $\begin{array}{l}\text { Formas ocupacionales del rol } \\
\text { productivo principal }\end{array}$ & 4 & 30 & 18 & 0 & 52 & 2.1 \\
\hline Formas ocupacionales de diversión & 7 & 26 & 9 & 8 & 50 & 2.0 \\
\hline Grupo social de la vida hogareña & 2 & 28 & 27 & 0 & 57 & 2.3 \\
\hline $\begin{array}{l}\text { Grupo social del rol productivo } \\
\text { principal }\end{array}$ & 6 & 22 & 24 & 0 & 52 & 2.1 \\
\hline \hline Grupo social de diversión & 11 & 22 & 9 & 0 & 42 & 1.7 \\
\hline $\begin{array}{l}\text { Espacios físicos, objetos y recursos en } \\
\text { la vida hogareña }\end{array}$ & 3 & 22 & 27 & 8 & 60 & 2.4 \\
\hline $\begin{array}{l}\text { Espacios físicos, objetos y recursos en } \\
\text { el rol productivo }\end{array}$ & 5 & 16 & 24 & 16 & 61 & 2.4 \\
\hline \hline $\begin{array}{l}\text { Espacios físicos, objetos y recursos de } \\
\text { la diversión }\end{array}$ & 6 & 16 & 30 & 4 & 56 & 2.2 \\
\hline
\end{tabular}

\section{" Primer análisis descriptivo del perfil ocupacional}

\section{Escala de Identidad Ocupacional}

La identidad ocupacional es cómo una persona piensa y se siente acerca de sí misma como un ser ocupacional ( $\underline{3})$.

Esto implica el autoconocimiento volitivo, es decir, el conocimiento que permite a una persona anticipar, elegir, experimentar e interpretar su comportamiento ocupacional. 
También las disposiciones (disfrutar, valorar y sentirse capaz de realizar una ocupación), así como las costumbres y autoconciencia (aquello que experimentamos al desempeñar una ocupación). Ambas constituyen lo que nos motiva hacia ciertas ocupaciones.

La autoconciencia incluye: identificación de roles, incluyendo los roles tradicionales de trabajador/estudiante, padre/madre y/o miembro de un grupo; y roles informales como por ejemplo, liderar un grupo, estar al cuidado de otros, ser hermano/a, entre muchos otros. Para aquellas personas que no tienen roles formales, los roles informales pasan a ocupar un lugar muy importante. Por otra parte, los roles reflejan la energía, motivación y compromiso asociados a una identidad ocupacional ( $\underline{3}$ ).

Los once reactivos que conforman la escala de Identidad ocupacional son los siguientes:

- Tiene metas personales y proyectos

- Identifica un Estilo de Vida Ocupacional

- Espera el éxito

- Acepta responsabilidades

- Valora habilidades v limitaciones

- Tiene compromisos y valores

- Reconoce identidades y obligaciones

- Tiene intereses

- Se sintió eficaz (pasado)

- Encontró sentido / satisfacción en su Estilo de Vida (pasado)

- Hizo elecciones ocupacionales (pasado)

En el OPHI-II, los ocho primeros reactivos pertenecen a la persona en el aquí y el ahora, mientras que los últimos tres reactivos se refieren al funcionamiento de la persona en el pasado.

Cabe señalar, sin embargo, que para efectos de este estudio, cada uno de los reactivos de las tres sub escalas fueron evaluados en relación a la historia ocupacional de la persona antes de su ingreso al programa de tratamiento y rehabilitación. Esto, dado que el objetivo central de la investigación es determinar si existe una historia de funcionamiento ocupacional disfuncional que permite explicar, en parte, el consumo de drogas. Es posible suponer que una vez que una persona ingresa a un programa e inicia un proceso de tratamiento y rehabilitación, la narrativa de su historia ocupacional irá variando en la medida que se vayan internalizando nuevas experiencias positivas, valores y otras pautas de comportamiento. Por ejemplo, no es poco frecuente escuchar a una persona en tratamiento que refiera que desde ahora quiere dedicarse a ser buen padre y esposo y a cumplir con sus responsabilidades. Sin embargo, al indagar por este aspecto antes del ingreso al programa nos encontramos con una persona que no logra cumplir con las responsabilidades asociadas al rol de esposo/padre debido a que su preocupación principal en ese momento es el consumo de drogas y las actividades asociadas a éste.

Por otra parte, el indagar los aspectos ocupacionales de la vida de la persona en el pasado nos permite identificar si existen algunas fortalezas en que la persona se puede apoyar y que serán de gran ayuda para el tratamiento en el presente. 
Recordemos, además que uno de los objetivos de este estudio es determinar si existen en el sujeto consumidor de drogas aspectos o patrones repetidos de comportamiento ocupacional que constituyan fortalezas o debilidades. El identificar estos aspectos positivos y negativos permitiría incluirlos en programas de prevención específica, que puedan evitar o retrasar la aparición del consumo.

En el presente estudio los promedios obtenidos en la Escala de Identidad Ocupacional oscilan entre 1.9 y 2.3, siendo el 2.1 el que se repite con mayor frecuencia. Este puntaje indica cierta disfunción ocupacional.

Si analizamos cada uno de los reactivos de esta sub escala por separado, encontraremos lo siguiente:

- El puntaje más bajo (1.9) lo obtienen dos reactivos: "espera el éxito" y "acepta responsabilidades". Esto implica que la persona tiene una dudosa capacidad para enfrentar obstáculos, limitaciones o fracasos; tiene inseguridad respecto a la posibilidad de obtener éxito; se afecta fácilmente cuando enfrenta desafíos. Por otra parte, tiende a evitar responsabilidades en acciones personales; culpa a otros por sus fracasos; se critica en exceso; tiende a negar o se siente abrumado por la retroalimentación.

- Los puntajes más altos (2.4 y 2.3) lo obtienen los reactivos "tiene intereses" y "valora habilidades y limitaciones". A pesar de ser los puntajes más altos obtenidos en esta sub escala, igualmente indican cierto grado de disfunción ocupacional. Por una parte, implica que a la persona se le dificulta identificar intereses; siente atracción, aunque a veces limitada, hacia ocupaciones que corresponden a sus habilidades; sus intereses no concuerdan con sus habilidades. Por otra parte, implica que la persona sobreestima o subestima sus capacidades, llevándola a desarrollar ocupaciones inadecuadas y se le dificulta compensar limitaciones con capacidades.

- El puntaje que más se repite (2.1) se obtiene en tres de un total de once reactivos que conforman la sub escala. Estos son: "identifica un estilo de vida ocupacional deseado", "tiene compromisos y valores", "reconoce identidades y obligaciones". Lo anterior implica que la persona tiene dificultades para identificar el estilo de vida ocupacional que desea; tiene fuertes dudas o insatisfacciones con la actividad ocupacional que desempeña; se le dificulta identificar cómo estructurar y organizar su tiempo y logra identificar una o más ocupaciones significativas. Por otra parte, en esta persona los valores en conflicto limitan la elección ocupacional; desconoce el objetivo o dirección en su vida; tiene valores no compartidos por uno o varios grupos sociales. Finalmente, a esta persona se le dificulta verse en uno o más roles; se compromete marginalmente con los roles; se le dificulta identificar responsabilidades de los roles a pesar de desearlos; se identifica en forma débil con los roles.

- El puntaje 2.2 es obtenido por los reactivos "se sintió eficaz" y "encontró sentido/ satisfacción en su estilo de vida". Esto implica que la persona sintió que la responsabilidad era inadecuada cuando se le dio; se sintió desalentada cuando se enfrentó a desafíos. Por otra parte, esta persona estaba de algún modo descontenta con sus roles de vida; tenía alguna dificultad para identificar intereses y tenía dificultad para encontrar sentido a su vida.

- El puntaje 2.0 es obtenido por los reactivos "tiene metas personales y proyectos" e "hizo elecciones ocupacionales". Esto implica que las metas o proyectos se encuentran por arriba o por debajo de las habilidades estimadas; la persona no está muy motivada para trabajar en metas o proyectos 
personales; su compromiso y motivación son limitados. Por otra parte, esta persona ha tenido dificultad para identificarse con un estilo de vida, hizo elecciones ocupacionales que interfirieron con la búsqueda de una historia de vida; su historia de vida la condujo a elecciones ocupacionales negativas.

\section{Escala de Competencia Ocupacional}

La competencia ocupacional es la capacidad que tiene una persona para actualizar o poner en práctica una identidad ocupacional de modo que produzca satisfacción para sí mismo y cubra las demandas del ambiente ( $\underline{3})$.

La competencia ocupacional contempla el mantener un patrón de rutina ocupacional que cubra roles, genere satisfacción, permita la expresión de intereses y conlleve además el cumplimiento de estándares personales.

Los nueve reactivos de esta escala son los siguientes:

- Mantiene un Estilo de Vida Satisfactorio

- Cumple con las expectativas de sus roles

- Trabaja hacia metas personales

- Cumple con los estándares de rendimiento personales

- Organiza su tiempo para cumplir responsabilidades

- Participa en Intereses

- Cumplió con sus Roles (pasado)

- Mantuvo Hábitos (pasado)

- Logró Satisfacción (pasado)

Al igual que en la escala anterior, los seis primeros reactivos corresponden a circunstancias actuales, mientras que los tres últimos se refieren al funcionamiento ocupacional en el pasado. Sin embargo, como ya se señaló, para este estudio todos los reactivos fueron evaluados en relación a las circunstancias anteriores al ingreso al programa de tratamiento y rehabilitación.

En el presente estudio los promedios obtenidos en la Escala de Competencia Ocupacional oscilan entre 1.7 y 2.2. Este puntaje estaría indicando entre cierta disfunción ocupacional hasta disfunción ocupacional propiamente tal.

Cabe señalar que esta es la sub escala que obtiene más bajos puntajes. Si analizamos cada uno de los reactivos por separado, encontraremos lo siguiente:

- El puntaje más bajo (1.7) lo obtiene el reactivo "cumplió con sus roles". Esto implica que la persona ha tenido dificultad para equilibrar las demandas de los roles; ha tenido un desenvolvimiento variable en sus roles; ha tenido roles conflictivos; en ocasiones ha experimentado fracasos en uno o más roles o ha tenido carencia de roles.

- El puntaje más alto (2.2) lo obtiene el reactivo "cumple con los estándares de rendimiento personales". A pesar de ser el puntaje más alto, el valor obtenido indica cierto grado de disfunción ocupacional. Lo anterior implica que ya sea debido a expectativas personales excesivas o debido a limitaciones significativas o a una capacidad disminuida, existe una continua diferencia entre resultados y estándares que generan una duda en sí mismo. 
- El puntaje que más se repite (2.0) lo obtienen tres reactivos: "cumple con las expectativas de sus roles", "mantuvo hábitos" y "participa en intereses". Lo anterior implica que esta persona experimenta una dificultad creciente para cumplir con las expectativas de sus roles y tiene pocas obligaciones que le permitan mantener un patrón consistente de logro. Por otra parte, esta persona ha mantenido una rutina diaria poco consistente y poco organizada de acuerdo a sus metas; también ha experimentado periodos de significativa desorganización en su vida diaria. Finalmente, esta persona tiene una participación inconsistente en intereses; tiene dificultad para participar en intereses importantes; su problema de salud limita su participación en intereses del pasado; tiene dificultad para encontrar y/o desarrollar nuevos intereses.

- El puntaje 1.8 lo obtienen los reactivos "trabaja hacia metas personales" y "mantiene un estilo de vida satisfactorio". Esto implica que la persona tiene dificultad para mantenerse enfocado hacia una o más metas; lucha por metas inalcanzables que lo llevan a fracasos constantes; sus metas han sido impactadas de manera significativa por su problema de salud; en ocasiones pierde el compromiso hacia sus metas. Por otra parte, esta persona tiene dificultades para mantener roles, proyectos personales o actividades; tiene dificultad para llenar su vida con roles o proyectos personales adecuados; mantiene un estilo de vida estresante con demasiadas demandas o prioridades; su estilo de vida muestra una clara falta de dirección o propósito; hay conflicto entre roles y responsabilidades.

- El puntaje 1.9 lo obtiene el reactivo "organiza su tiempo para cumplir responsabilidades". Esto significa que la persona tiene grandes dificultades en organizar rutinas que le permitan cubrir múltiples responsabilidades y adaptarse a los cambios. En ocasiones es incapaz de adaptar su rutina a nuevas circunstancias o de organizar una rutina que le permitan cubrir con labores básicas de autocuidado. Muchas veces su rutina es expresión de conductas altamente desadaptadas, como el consumo de drogas.

- El puntaje 2.1 lo obtiene el reactivo "logró satisfacción". Esto implica que la persona se ha sentido insatisfecha con su estilo de vida; no ha logrado equilibrar las actividades de autocuidado, trabajo y diversión; ha tenido fracasos importantes que interfirieron en sus logros; perdió un interés o meta importante sin reemplazarla; tuvo dificultades para dar seguimiento a sus metas.

\section{Escala de Ambientes de Comportamiento Ocupacional}

Los Ambientes de Comportamiento Ocupacional son los ambientes diarios en los que la persona vive (hogar o vivienda). Incluye el rol más productivo de su vida (escuela, lugar de trabajo) y también involucra la diversión (barrio, hogar y lugares de esparcimiento tales como teatros, cines, parques, gimnasios, entre otros).

Los Ambientes de Comportamiento Ocupacional miden el impacto del medio ambiente sobre la vida ocupacional del cliente ( $\underline{3})$.

Los nueve reactivos de esta escala son los siguientes:

- Formas ocupacionales de vida en el hogar

- Formas ocupacionales del rol productivo principal

- Formas ocupacionales de diversión

- Grupo social de vida hogareña 
- Grupo social del rol productivo principal

- Grupo social de diversión

- Vida del Hogar. Espacio físico, objetos, recursos.

- Rol productivo principal. Espacio físico, objetos, recursos.

- Diversión. Espacio físico, objetos, recursos.

En el presente estudio los promedios obtenidos en la Escala de Competencia Ocupacional oscilan entre 1.7 y 2.4. Este puntaje estaría indicando entre cierta disfunción ocupacional hasta disfunción ocupacional propiamente tal.

Cabe señalar que es en esta escala en la que se obtienen los puntajes más altos. Si analizamos cada uno de los reactivos por separado, encontraremos lo siguiente:

- El puntaje más bajo (1.7) lo obtiene el reactivo "grupo social de diversión". Esto implica que la baja o excesiva demanda de interacción o colaboración limitan el funcionamiento de la persona; el ambiente emocional y práctico retraen su funcionamiento; los otros no reconocen sus habilidades, contribuciones y esfuerzos y muchas veces se siente incapaz de influir en sus capacidades.

- El puntaje más alto (2.4) lo obtienen los reactivos "espacio físico, objetos y recursos de la vida hogareña" y "espacio físico, objetos y recursos del rol productivo". Lo anterior implica que la persona percibe el medio ambiente y objetos de su hogar y rol productivo principal de cierta manera accesibles; inseguros; de cierta manera invasivos e incómodos; de cierta manera desmotivantes o faltos de significado; en algún grado no le prestan apoyo.

- El puntaje 1.9 lo obtiene el reactivo "formas ocupacionales de vida en el hogar". Ello implica que las demandas y/o oportunidades de la vida en el hogar de alguna manera no congrúen con intereses y habilidades tanto físicas, como cognitivas y emocionales. Por otra parte, el tiempo y esfuerzo requeridos ocasionalmente son apropiados al tiempo y energía disponibles.

- El puntaje 2.0 lo obtiene el reactivo "formas ocupacionales de diversión". Al igual que en la descripción anterior, las demandas y/o oportunidades de diversión de alguna manera no congrúen con intereses y habilidades tanto físicas, como cognitivas y emocionales. Por otra parte, el tiempo y esfuerzo requeridos ocasionalmente son apropiados al tiempo y energía disponibles.

- El puntaje 2.1 lo obtienen los reactivos "formas ocupacionales del rol productivo principal" y "grupo social del rol productivo principal". Al igual que en la descripción anterior, las demandas y/o oportunidades del rol productivo principal de alguna manera no congrúen con intereses y habilidades tanto físicas, como cognitivas y emocionales. Por otra parte, el tiempo y esfuerzo requeridos ocasionalmente son apropiados al tiempo y energía disponibles. Además, en cuanto al rol productivo principal la baja o excesiva demanda de interacción limita su funcionamiento; el ambiente emocional y práctico retraen su funcionamiento; percibe que los otros no reconocen sus habilidades, contribuciones y esfuerzos.

- El puntaje 2.2 lo obtiene el reactivo "espacio físico, objetos y recursos de diversión". Lo anterior implica que la persona percibe el medio ambiente y objetos de diversión de cierta manera accesibles, pero inseguros; de cierta manera invasivos e incómodos; de cierta manera desmotivantes o faltos de significado; en algún grado no le prestan apoyo.

- El puntaje 2.3 lo obtiene el reactivo "grupo social de la vida hogareña". Esto implica que la baja o excesiva demanda en el hogar de interacción o colaboración limitan el funcionamiento de la persona; el ambiente emocional y práctico retraen su funcionamiento; los otros no reconocen sus habilidades, 
contribuciones y esfuerzos y muchas veces se siente incapaz de influir en sus capacidades.

\section{Primera discusión hallazgos de la investigación}

Los primeros hallazgos de este estudio indican que el sujeto consumidor de drogas que se encuentra en un programa de tratamiento y rehabilitación presenta una historia ocupacional disfuncional.

Esta disfunción se encuentra tanto en la identidad, como la competencia ocupacional y los ambientes de comportamiento ocupacional.

Si bien todos los ámbitos del desempeño ocupacional se encuentran con algún grado de disfunción o alteración, es posible identificar en cada escala aspectos que son más disfuncionales que otros y sobre los que se debería poner especial atención, y aspectos que se encuentran menos alterados que otros y que se debieran potenciar. Veamos.

\section{Escala de Identidad Ocupacional:}

- La mayor dificultad que presenta el sujeto en esta escala es la capacidad para enfrentar obstáculos y la tendencia a culpar a otros por sus fracasos. Se trata, por lo tanto, de una persona con baja tolerancia a la frustración y con dificultad para hacerse cargo de sus responsabilidades y su conducta. Tiende a poner la responsabilidad de sus actos fuera de su persona lo que dificulta la toma de conciencia y la disposición para el cambio.

- La mayor fortaleza es que en ocasiones logra identificar uno o más intereses, valorar algunas capacidades y reconocer algunas limitaciones. Estas características son posibles de abordar y potenciar en una intervención.

\section{Escala de Competencia Ocupacional:}

- La mayor dificultad que enfrenta el sujeto en esta escala es el cumplimiento de sus roles, lo que significa que no sólo tiene dificultad para cumplir con las demandas de sus roles, sino que muchas veces enfrenta roles conflictivos o contradictorios. Se trata de una persona que presenta dificultad para identificarse con uno o más roles $y$, muchas veces, a pesar de identificarse, no logra cumplir con las demandas que éstos implican, lo que puede producir sentimientos de frustración y ansiedad.

- La mayor fortaleza en esta escala es que en ocasiones el sujeto logra cumplir con estándares personales. Sin embargo, el grado de disfunción que presenta indica que la mayor parte del tiempo estos estándares no se alcanzan, ya sea porque sobreestima sus capacidades o porque adolece de graves limitaciones. En una intervención es posible abordar estos aspectos buscando acercar capacidades con limitaciones objetivas del individuo, favoreciendo el desarrollo de acciones más congruentes con su realidad.

- Recordemos que esta escala es la que presenta más bajos puntajes, es decir que lo más afectado en esta persona es su capacidad para poner en práctica una identidad ocupacional, con satisfacción para sí mismo y cubriendo las demandas del ambiente. 
Escala de Ambientes de Comportamiento Ocupacional:

- La mayor dificultad que enfrenta el sujeto en esta escala es el grupo social de diversión. Esto implica que, ya sea por baja o por alta demanda de interacción el individuo se ve limitado en su funcionamiento, siente que los otros no reconocen sus esfuerzos, ni sus contribuciones y siente que no puede influir sobre sus capacidades.

- La mayor fortaleza del individuo en esta escala son los espacios físicos, objetos y recursos de la vida hogareña y del rol productivo principal. Esto significa que el individuo tiene la percepción de tener acceso a lo necesario para desenvolverse, aunque en ocasiones estos objetos se perciban como inseguros o faltos de significado.

- Lo anterior indica que se debe considerar como parte de las medidas de prevención del consumo de drogas una intervención sobre el ambiente en que se desenvuelve el sujeto, tanto para potenciar los factores que facilitan o potencian su funcionamiento, como para controlar aquellos que lo dificultan o alteran. No debemos olvidar que para lograr la motivación de una persona, para que ésta entre en "flujo" es necesario que la actividad y ambiente sean concordantes con sus habilidades e intereses, que no estén ni muy por encima, ni muy por debajo de éstas, porque esto provocará la desmotivación y no participación.

- La mayor parte de los programas preventivos actualmente en curso ponen el énfasis en los cambios y actitudes personales y no consideran la intervención sobre el ambiente. El Modelo de Ocupación Humana propone considerar ambos aspectos al momento de planificar una intervención (sujeto en interacción con sus medios ambientes).

Considerando los aspectos más afectados, podemos concluir que esta persona ha tenido pocas oportunidades a lo largo de su vida de realizar actividades con propósito y significado para sí mismo (ocupaciones) que le permitan desarrollar destrezas, cumplir demandas ambientales (competencia), obtener satisfacción personal y así adaptarse al ambiente, con un sentido de satisfacción.

Su historia ocupacional se caracteriza por un alto grado de dificultad para equilibrar actividades de autocuidado, productividad y tiempo libre. Tampoco logra satisfacción con las actividades que realiza y la mayor parte de las veces, éstas tienen poco significado para sí mismo.

Lo anterior lo convierte en una persona vulnerable, con alto riesgo de presentar conductas desadaptadas, entre las cuales se encuentra el consumo de drogas.

Por ello se vuelve relevante conocer, evaluar y posteriormente intervenir el desempeño ocupacional de una persona en etapas tempranas, como parte de un programa de prevención que pretenda ser exitoso en evitar el inicio del consumo de drogas.

\section{- Referencias bibliográficas}

1 UNIVERSIDAD DE CHILE, FACULTAD DE MEDICINA, ESCUELA DE SALUD PÚBLICA (2002). "Evaluación de proceso de las instituciones en convenio 
con CONACE-FONASA en programas de apoyo a los planes de tratamiento y rehabilitación en drogas; y evaluación de resultados de los programas en los usuarios de instituciones en convenio".

2 RIVEROS, M.E. "Historia de desempeño ocupacional en usuarios de programas de tratamiento y rehabilitación en drogas: una propuesta para la prevención". Tesis para optar al grado de Magíster en Salud Pública. Escuela de Salud Pública. Facultad de Medicina. Universidad de Chile, 2003.

3 DE LAS HERAS, C.G. (2001). Manual "Rehabilitación y Vida: Modelo de Ocupación Humana". Producido por Reencuentros. Centro de Rehabilitación Psicosocial Américo Palma Ltda. Santiago, Chile.

4 UIC/University of Illinois at Chicago. ITO/Instituto de Terapia Ocupacional. (1998) Manual del Usuario de la Entrevista de Historia del Desempeño Ocupacional (versión 2.0) OPHI-II. Capítulo 1.

5 KIELHOFNER, G. (1995). A Model of Human Occupation. Theory and Apliccation. $2^{\text {nd }}$ Edition. Baltimore, MD Williams \& Wilkins.

6 BECOÑA IGLESIAS, ELISARDO (1999). Bases Teóricas que sustentan la prevención en drogas. Capítulos 1 y 2 Plan Nacional sobre drogas. Universidad de Santiago de Compostela. Madrid, 1999. Madrid.

7 COMAS, D., ESPÍN, MARTÍN, M. Editores (1992). Fundamentos Teóricos en Prevención de Drogodependencias. Grupos Interdisciplinar sobre Drogas. Ed. Fundamentos. Madrid, España.

8 CONACE - Consejo Nacional para el Control de Estupefacientes. República de Chile. Ministerio del Interior (1998). Programa de Especialización en Desarrollo Personal Preventivo. Módulo II: Una opción paradigmática y una propuesta metodológica para realizar estudios epidemiológicos del consumo de drogas en países de América Latina. Claudio Molina y Francisco Rojas.

9 CONACE - Consejo Nacional para el Control de Estupefacientes. República de Chile. Ministerio del Interior (1996). Drogas. Conceptos Básicos y herramientas para la Prevención.

10 FOSSEY, E. (1996). Using the Occupational Performance History Interview (OPHI). Therapists reflections. British Journal of Occupational Therapy, 59 (5).

11 KIELHOFNER, G. \& BURKE, J. (1980). A Model of Human Occupation. Part One: conceptual framework and content. American Journal of Occupational Therapy, 34.

12 KIELHOFNER, G. \& HENRY, A.D, (1988). Development and investigation of the occupational performance history interview. American Journal of Occupational Therapy, 42

13 MALLINSON, T., KIELHOFNER, G. \& MATTINGLY, C. (1996). Metaphor and meaning in a clinical interview. American Journal of Occupational Therapy, $50(5)$.

14 MALLINSON, T. (In Press). Construct validity of the OPHI (revised). Canadian Journal of Occupational Therapy. 